\author{
Marquette University \\ e-Publications@Marquette
}

College of Nursing Faculty Research and

Publications

Nursing, College of

$9-2013$

\title{
A Review of Patients with Renal Disease Undergoing Vascular Access Surgery: Is Gray-scale Ultrasound Enough?
}

Nancy M. Raml

Marquette University

Susan Breakwell

Marquette University, susan.breakwell@marquette.edu

Follow this and additional works at: https://epublications.marquette.edu/nursing_fac

Part of the Nursing Commons

\section{Recommended Citation}

Raml, Nancy M. and Breakwell, Susan, "A Review of Patients with Renal Disease Undergoing Vascular Access Surgery: Is Gray-scale Ultrasound Enough?" (2013). College of Nursing Faculty Research and Publications. 307.

https://epublications.marquette.edu/nursing_fac/307 
Marquette University

e-Publications@Marquette

\title{
Nursing Faculty Research and Publications/College of Nursing
}

This paper is NOT THE PUBLISHED VERSION; but the author's final, peer-reviewed manuscript. The published version may be accessed by following the link in the citation below.

Journal of Vascular Nursing, Vol. 31, No. 3 (September 2013): 111-117. DOI. This article is (C) Elsevier and permission has been granted for this version to appear in e-Publications@Marquette. Elsevier does not grant permission for this article to be further copied/distributed or hosted elsewhere without the express permission from Elsevier.

\section{A Review of Patients with Renal Disease Undergoing Vascular Access Surgery: Is Gray- Scale Ultrasound Enough?}

\author{
Nancy M. Raml \\ Department of Vascular Surgery, Zablocki VA Medical Center and Marquette University College of \\ Nursing, Milwaukee, Wisconsin \\ Susan Breakwell \\ Institute for End of Life Care Marquette University, College of Nursing, Milwaukee, Wisconsin
}

\section{Abstract \\ Background}

An adequate fistula or graft is essential to long-term survival and quality of life for patients with endstage renal disease (ESRD) who are receiving hemodialysis because of its lower complication rates, lower costs, and prolonged patency. Use of duplex ultrasound for preoperative planning is currently recommended by the Kidney Disease Outcomes Quality Initiative for patients with ESRD with prior fistulas or grafts, central lines, pacemakers, and prior chest or arm surgery. The preoperative 
evaluation consisted of gray-scale ultrasound and physical examination in all patients with ESRD in this study. The current study determined the baseline data, including the type of vascular access, functional patency of access, associated morbidity, and preoperative demographics and comorbidities, including prior dialysis access. The primary objective was to determine the frequency of revision surgery, to identify the potential cases that may indicate the need for better assessments (eg, duplex ultrasound), and to improve fistula and graft success rates.

\section{Materials and methods}

A retrospective chart review of patients with ESRD who underwent native fistula or graft access creation in a 13-month time period from 2010 to 2011 was completed. Seventy-six surgical procedures were performed on 53 subjects. Included variables were age, race, gender, smoking status, body mass index, stage of chronic kidney disease at referral, previous central lines/pacemakers, fistulas, or grafts. Comorbidities identified included diabetes mellitus (DM), hypertension (HTN), and coronary artery disease (CAD). The types of access, location, maturation, infection, failure, or revision were noted. Continuous variables are shown in frequencies and mean. Categoric data were compared using chisquare analysis.

\section{Results}

During the 13-month study period, 76 surgical procedures were performed in 53 patients, with $39.6 \%$ of patients undergoing multiple surgical procedures. The majority of patients were male (98\%) and white (58.5\%), with a history of HTN (96.2\%) and DM (64.2\%). The mean age was 68 years, with most patients presenting in stage 5 chronic kidney disease (92.5\%). Some $67.9 \%$ of patients had prior central lines or pacemakers; of those, $56.6 \%$ had previous fistulas or grafts. Negative significance was determined between the comorbidities DM/HTN/CAD alone or grouped as a cohort and multiple surgeries. Positive significance was found between multiple surgical procedures and those with prior access/pacemaker/central line (chi-square [1, $N=53]=$ exact $P=.04$ ).

\section{Conclusions}

Patients with ESRD undergoing access creation presenting with prior central lines, pacemakers, or arm surgery (fistulas or grafts) were more likely to undergo multiple surgeries to obtain a functional graft or fistula for hemodialysis use than those patients with ESRD without prior central lines, pacemakers, or arm surgery (fistulas or grafts). Color duplex ultrasound should be considered as a standard for preoperative assessment in an effort to improve fistula or graft success rates.

End-stage renal disease (ESRD) is the culmination of progressive chronic kidney disease (CKD). Patients with ESRD often begin hemodialysis with 1 of 3 types of access: central venous catheters, synthetic arteriovenous (AV) grafts, or native arteriovenous fistulas (AVFs). Central venous catheters are the least desirable of the access types because of the potential of infection. They generally should be used for emergency or temporary use. ${ }^{1}$ The AV graft access is made of polytetrafluoroethylene material linking the artery to a vein. The AVF uses the patient's own vein and artery. AVFs have longer durability than synthetic grafts and often have fewer complications. ${ }^{2}$ A properly functioning fistula is a lifeline to patients with ESRD who are receiving hemodialysis. Careful preoperative planning and selection of type and location of access are imperative to a successful outcome. Guidelines from the National Kidney 
Disease Outcomes Quality Initiative (KDOQI) recommend creation of an AVF as the access of choice for patients with ESRD before initiating hemodialysis. Also included in the recommendations is the use of duplex ultrasound examination before AVF or AV graft placement in those patients with previous neck, arm, or chest surgery; current or previous central catheters; current or previous pacemakers; or edema or collateral vein development. ${ }^{3}$ Prior central or peripheral venous punctures can cause scarring to vessels that interfere with dilation of the vein, causing turbulent blood flow and predisposing the fistula to stenosis. ${ }^{4}$

AVF creation is largely dependent on vein and artery anatomy, with physical examination able to identify grossly acceptable veins with assistance of a tourniquet. The pulse quality and elasticity of the radial artery, and to a lesser extent the brachial artery because of its anatomic depth, may be palpable. Additional consideration must be given to the varied arterial and venous anatomy that cannot be visualized or palpated by physical examination alone. Malovrh ${ }^{5}$ demonstrated that veins detected by physical examination alone were found only $46.5 \%$ of the time, and veins that were present but not visible to the examiner were found $53.5 \%$ of the time.

Duplex ultrasound is the most cost-effective noninvasive test for dialysis access planning. The study combines B-mode (black and white brightness or gray scale), which identifies structural dimensions of vessel anatomy, and adds color scale duplex, which displays blood blow velocity and direction within the vessel being studied. ${ }^{6}$ Planken et $\mathrm{al}^{7}$ studied repetitive assessment of superficial venous diameters with B-mode only ultrasound using a blood pressure cuff or tourniquet. They found significant variability in forearm superficial venous diameters between subjects and time of examination, also indicating the importance of including arterial evaluation in the workup.

For this project, a literature review was undertaken and a retrospective chart review of patients with ESRD was conducted. The patients with ESRD who were referred to the surgeons at the study site medical center's vascular clinic were examined by the surgical providers. There was no formal preoperative protocol. The examination consisted of gray-scale ultrasound and physical examination to identify target vessels for use in access creation. A surgical plan was formulated from this workup. Duplex ultrasound was not part of the preoperative workup.

\section{Literature review and synthesis}

The purpose of this literature review was to identify the best evidence to support a preoperative protocol development for patients with ESRD. The appraisal of evidence was evaluated using the Johns Hopkins nursing evidence-based practice appraisal tools. These tools gauge the strength of the recommendations found in the studies or articles with levels 1 to 5 . Level I indicates the strongest evidence, and level $V$ indicates the weakest. The quality is graded with an $A$ to $C$ system. Grade $A$ is high, B is good, and C is low with major flaws. ${ }^{8}$ A total of 11 studies or articles were selected and appraised. The majority of articles were qualitative or meta-synthesis studies, with 1 meta-analysis and 4 quasi-experimental studies. The strength of the evidence ranged from level 1 to level 3, with the quality of evidence grade $B$ (Table 1$)$.

Table 1. Summary of research findings 


\begin{tabular}{|c|c|c|c|}
\hline $\begin{array}{l}\text { Strength of } \\
\text { the Evidence }\end{array}$ & $\begin{array}{l}\text { No. of } \\
\text { Studies }\end{array}$ & Summary of Findings & $\begin{array}{l}\text { Quality of the } \\
\text { Evidence }\end{array}$ \\
\hline Level 1 & 1 & & $\mathrm{~B}$ \\
\hline Glass et $\mathrm{al}^{10}$ & & $\begin{array}{l}\text { Duplex ultrasound is important and should be used } \\
\text { routinely in radiocephalic fistula creation. }\end{array}$ & \\
\hline Level 2 & 4 & & \\
\hline Allon et al ${ }^{14}$ & & $\begin{array}{l}\text { Routine preoperative vascular mapping with sonography } \\
\text { results in a significant increase in placement of AVFs and } \\
\text { improves the likelihood of a successful fistula. }\end{array}$ & $B$ \\
\hline Ku et $\mathrm{al}^{16}$ & & $\begin{array}{l}\text { High-resolution ultrasonography effective tool in } \\
\text { measuring radial artery intima-media thickness in } \\
\text { uremic patients before AVF operation. }\end{array}$ & B \\
\hline Malovrh4, 5 & & $\begin{array}{l}\text { Duplex sonography may provide useful data on } \\
\text { preoperative morphologic and functional characteristics } \\
\text { of vessels used for AVF construction and is helpful in } \\
\text { patients with inadequate clinical vein visibility. }\end{array}$ & $B$ \\
\hline $\begin{array}{l}\text { Planken } \\
\text { et } \text { al }^{7}\end{array}$ & & $\begin{array}{l}\text { Ultrasound venous diameter assessments are } \\
\text { comparable to a tourniquet versus graduated pressure } \\
\text { cuff. Variations in venous diameters should be taken } \\
\text { into account before vascular access surgery. }\end{array}$ & $B$ \\
\hline Level 3 & 6 & & \\
\hline $\begin{array}{l}\text { Grogan } \\
\text { et } \mathrm{al}^{12}\end{array}$ & & $\begin{array}{l}\text { Critical stenosis was found in patent primary AVFs using } \\
\text { color Doppler ultrasound. }\end{array}$ & $B$ \\
\hline Ives et al ${ }^{13}$ & & $\begin{array}{l}\text { Preoperative vessel mapping provides useful } \\
\text { information regarding the choice of AVF. }\end{array}$ & $B$ \\
\hline Korten et al ${ }^{15}$ & & $\begin{array}{l}\text { Preoperative ultrasound measurement of radial arteries } \\
\geq 2.1 \mathrm{~mm} \text { and } \leq 2.5 \mathrm{~mm} \text { has the highest patency rate in } \\
\text { radiocephalic AVFs. }\end{array}$ & $\mathrm{B}$ \\
\hline $\begin{array}{l}\text { Lauvao } \\
\text { et } \mathrm{al}^{17}\end{array}$ & & $\begin{array}{l}\text { Duplex ultrasound-measured vein size is the major } \\
\text { predictor for a successful AVF. }\end{array}$ & $B$ \\
\hline $\begin{array}{l}\text { Roca-Tey } \\
\text { et } \text { al }^{18}\end{array}$ & & $\begin{array}{l}\text { Blood flow of the radial artery determined by color } \\
\text { Doppler ultrasonography is paramount for achieving } \\
\text { radiocephalic permeability and maturation after } \\
\text { creation of AVF creation. }\end{array}$ & $B$ \\
\hline $\begin{array}{l}\text { Salman } \\
\text { et al }{ }^{11}\end{array}$ & & $\begin{array}{l}\text { Ultrasound has a high degree of accuracy in detecting } \\
\text { inflow stenosis of AVFs when compared with } \\
\text { angiography. }\end{array}$ & $B$ \\
\hline
\end{tabular}

$\mathrm{AVF}=$ arteriovenous fistula.

Initial databases searched were PubMed and CINAHL. Both keyword and MeSH search terms were applied. Specific journals, such as The Journal of Vascular Access, were hand-searched for usable articles. Fifty-four articles were identified as potentially useful. Of those, 10 were identified as targeting the predominately male population at the study site medical center while addressing the specific topic of duplex ultrasound in the management of adult patients with ESRD with AVF. 
CINAHL or the Cumulative Index to Nursing and Allied Health provides information relating to nursing and allied health by using more than 1200 journals. ${ }^{9}$ The database was accessed to add a nursing perspective. Most dialysis access employees who work with patients with ESRD on hemodialysis are nurses. The search terms arteriovenous fistula, preoperative care, renal disease, kidney disease, ultrasonography, and surgical were applied to the CINAHL search. The initial obtained literature was then hand-searched, and reference lists were checked for additional resources. There were 7 articles accessed from this database. Of these, 1 article was chosen as relevant to duplex ultrasound and adult patients on renal dialysis. Three sources that were repeatedly referenced while hand-searching were acquired and reviewed for relevance. The majority of the sources were dated from 2006 to 2010. Eleven studies were selected as meeting the specific topic of duplex ultrasound in the management of adult patients with ESRD with AVF.

\section{Analysis of literature}

Although the majority of the research was level 3 and quality $B$, the evidence points to the importance of using duplex ultrasound as part of the routine preoperative planning for AVFs.4, 10, 11, 12, 13, 14, 15 Duplex ultrasound also is beneficial in fistula surveillance in the postoperative period.16, 17, 18 The level 1 meta-analysis by Glass et al ${ }^{10}$ included 433 patients who had duplex ultrasound as part of their preoperative evaluation. Findings showed that duplex ultrasound not only revealed optimum diameters for arterial and venous vessels but also detected anatomic variations, compressibility, and flow velocities, which resulted in nearly doubling the success rate of fistula placement. ${ }^{10} \mathrm{~A}$ recent study by Salman et $\mathrm{l}^{11}$ compared angiography, the gold standard for detecting inflow stenosis in fistulas, with ultrasound and found that ultrasound has a sensitivity of $91 \%$, specificity of $98 \%$, and positive and negative predictive values of $98 \%$ and $90 \% .{ }^{1}$ The studies reviewed also support duplex ultrasound use in the postoperative period to identify potential stenotic areas that may progress to thrombosis and failure of the AVF by identifying peak systolic velocity and systolic velocity ratio that increase in the presence of stenosis.11, 12, 13 Ives et al ${ }^{13}$ found that duplex ultrasound had $100 \%$ sensitivity and $85 \%$ specificity for postoperatively detecting a maturing fistula.

Duplex ultrasound is beneficial in measuring arterial and venous diameters, which are major predictors in fistula maturation. It was also effective in measuring radial artery vessel thickness, which when increased is associated with early fistula failure.5, 14, 15, 16, 17 Radial artery blood flow determined by color Doppler ultrasonography is paramount for achieving successful permeability and maturation in fistulas using the radial artery. ${ }^{18}$ Planken et al $^{7}$ found that repeated assessment of superficial venous diameter with B-mode only ultrasound using a blood pressure cuff or tourniquet to dilate the vessel showed that forearm superficial venous diameters vary substantially between subjects and time of examination, and point to the importance of including arterial evaluation in the preoperative workup. ${ }^{7}$

\section{Theoretic framework}

Evidence-based models have been developed to move evidence into practice. Many of the models share common phases, such as identifying clinical or potential problems, gathering best evidence, and appraising and evaluating potential change in practice, with implementation and evaluation. ${ }^{19}$ In nursing, the best evidence uses findings from research that are methodologically appropriate, rigorous, and clinically relevant. The intervention must be effective, using reliable and precise nursing assessments while considering the nature of the patient experience. ${ }^{20}$ The lowa Model of Evidence- 
Based Practice guides clinicians (physicians, nurses, allied health care) in the use of evidence to improve healthcare outcomes. It is based on a planned action process that includes conduct of research, use of current research, and other types of evidence, such as case reports, expert opinion, scientific principles, and theory. ${ }^{21}$ The problem or knowledge focus trigger is the initial step, along with determining whether the trigger is a priority for the organization. ${ }^{21}$ The lowa Model illustrates a pathway to promote quality patient care and problem solving by incorporating a team approach in which to ensure a safe decision-making process on which to base change. ${ }^{22}$ Adequate preoperative planning is essential for successful maturation and use of arterial venous fistula or graft creation. Current practice at the study site medical center does not use duplex ultrasound. The lowa Model includes an emphasis on the organizational process, involving collaboration of various team members. It is a sound model to use while exploring and implementing practice change in the preoperative workup of patients on dialysis.

\section{Purpose}

The primary objective of the study was to determine whether the preoperative workup for patients who are receiving dialysis is adequate. This chart review will identity the frequency of revision surgery.

\section{Materials and methods}

\section{Study design}

A chart review of all adult male and female patients who consecutively underwent fistula or graft creation surgery in a 13-month period from 2010 to 2011 were retrospectively identified through surgical procedural records. These represented the patients of the 2 surgeons who perform vascular access surgery at the study site. The database was Health Insurance and Portability Act compliant. The study was approved by the human research committees at both the study site medical center and Marquette University. All data were deidentified and collected by the primary investigator at the study site medical center. Baseline demographics, including age, gender, race, body mass index (BMI), smoking status (within 1 year of surgery), and stage of CKD at the time of surgery were extracted via electronic medical records. Comorbidities collected were diabetes mellitus (DM), HTN, and coronary artery disease (CAD). Preexisting fistula or grafts, central lines, or pacemakers were identified. The type of surgical procedures performed, grafts or fistulas, and their placement in the arm or leg were collected. Additional complications, such as infection, failure of fistula to mature (dilation of the vein to 6-8 $\mathrm{mm}$ ), graft removal, or fistula ligation, were documented if they occurred within the study period. Functional use of the access, whether graft or fistula within the study period, was not accurately identified. All patients underwent physical examination and gray-scale ultrasound during the preoperative planning.

\section{Statistical analysis}

Seventy-six surgical procedures were performed in 53 subjects during the 13-month study period between 2010 and 2011. Twenty-one (39.6\%) of the subjects underwent multiple surgical procedures during the study period. Seventeen subjects had 2 procedures, 3 subjects had 3 procedures, and 1 subject had 4 surgical procedures within the study period. These 21 subjects were identified as duplicated census. Baseline characteristics were summarized with continuous and categoric variables. Continuous variables such as age were categorized as frequencies in decades and mean age. BMI data 
were recorded as $\geq 30$ or $<30 \mathrm{~kg} / \mathrm{m}^{2}$ and mean. Chi-square analysis was performed to determine any association between multiple surgeries and previous access with prior grafts or fistulas, central lines, or pacemakers. A formal power analysis was conducted using 0.80 power and an alpha error probability of 0.05. A large effect with a sample size of 32 was obtained. ${ }^{23}$ Data were analyzed using the Statistical Package for the Social Sciences version 19.0 (SPSS, Inc, Chicago, III).

\section{Results}

The study cohort included 53 subjects with late-stage CKD, including $92.5 \%$ presenting in stage 5 and $7.5 \%$ presenting in stage 4 . Most subjects were male, with $1.9 \%$ representing the female gender, which is representative of the study center's population. A majority of the subjects were white (58.5\%), with the remainder being African American (41.5\%). There were no Hispanics or Asian/Pacific Americans identified. Smoking status was examined with more subjects identified as nonsmokers within 1 year of surgery.

Consistent with the most common causes of CKD being DM and HTN, $64.2 \%$ of subjects were diabetic and $96.2 \%$ had HTN. The BMI was representative of the majority of subjects being in stage 5 CKD, in whom weight loss is common. Only $30.2 \%$ of subjects had prior CAD (Table 2). Approximately $40 \%$ of subjects had multiple surgical procedures in the study time frame, with 1 subject undergoing 4 procedures in 1 year. A substantial percentage of subjects (67.9\%) had prior central lines or pacemakers before surgery, with more than half (56.6\%) having had a past fistula or graft before the study time period (Table 3). Of the 53 subjects, 21 were identified as undergoing multiple procedures for fistula or graft creation during the 13-month study period. Forty subjects from the total sample had prior access with a central line, fistula, or graft. Chi-square analysis showed an association between those with multiple surgeries in the study period and those with a prior access. Subjects who had access preoperatively were more likely to undergo multiple surgeries within 13 months to obtain a functional graft or fistula for hemodialysis use. This relationship was statistically significant: chi-square $(1, N=53)=$ exact $P=.04$. Complications such as infection and failure to mature, with or without subsequent graft removal or fistula ligation, occurred in $21 \%$ of patients. Maturation of fistulas and availability of grafts would require a prospective analysis and will be addressed in the "Discussion" section. The Centers of Medicare and Medicaid Services national initiative Fistula First has set goals of increasing the use of AVFs to $\geq 50 \%$ for new patients and $\geq 66 \%$ for those already on hemodialysis. According to this initiative, only approximately $41 \%$ of patients on hemodialysis nationwide dialyze with a fistula. ${ }^{24}$ The precise percentage of grafts or fistulas available for functional use for hemodialysis was unable to be obtained because of the retrospective nature of the study, although $21 \%$ of subjects identified with complications during the study period would not qualify as having functional access for hemodialysis.

Table 2. Demographics of 53 subjects with chronic kidney disease undergoing access surgery

\begin{tabular}{|l|l|l|}
\hline Variable & $\mathbf{n}$ & $\mathbf{\%}$ \\
\hline Smoking & 19 & 35.8 \\
\hline DM & 34 & 64.2 \\
\hline HTN & 51 & 96.2 \\
\hline CAD & 16 & 30.2 \\
\hline
\end{tabular}




\begin{tabular}{|l|l|l|}
\hline $\mathrm{BMI} \geq 30 \mathrm{~kg} / \mathrm{m}^{2}$ & 22 & 41.5 \\
\hline $\mathrm{BMI}<30 \mathrm{~kg} / \mathrm{m}^{2}$ & 31 & 58.5 \\
\hline $\mathrm{Mean} \mathrm{BMI}$ & 28.9 & \\
\hline $\mathrm{CKD}$ & & \\
\hline Stage IV & 4 & 7.5 \\
\hline Stage V & 49 & 92.5 \\
\hline Total & 53 & 100.0 \\
\hline
\end{tabular}

$\mathrm{BMI}=$ body mass index; $\mathrm{CAD}=$ coronary artery disease; $\mathrm{CKD}=$ chronic kidney disease; $\mathrm{DM}=$ diabetes mellitus; HTN = hypertension.

Table 3. History of grafts/fistulas: Central lines or pacemakers in 53 subjects undergoing access surgery

\begin{tabular}{|l|l|l|}
\hline Variable & $\mathbf{n}$ & $\mathbf{\%}$ \\
\hline Graft & 5 & 9.4 \\
\hline Fistula & 19 & 35.8 \\
\hline Both & 6 & 11.3 \\
\hline Total* & 30 & 56.6 \\
\hline CL or PM & 36 & 67.9 \\
\hline Total & 53 & 100.0 \\
\hline
\end{tabular}

$\mathrm{CL}=$ central line; $\mathrm{PM}=$ pacemaker.

*Having fistula or graft before study.

\section{Discussion}

The KDOQI recommends duplex ultrasound for patients with prior neck, arm, or chest surgery; current or previous central catheters; current or previous pacemakers; edema or collateral vein development. ${ }^{3}$ Prior central or peripheral venous punctures to the veins cause scarring to the vessel that interferes with dilation of the vein, causes turbulent blood flow, and predisposes the fistula to stenosis. ${ }^{4}$ This review identified a substantial percentage of subjects (67.9\%) with prior central lines or pacemakers, with more than half $(56.6 \%)$ having had a past fistula or graft. Those patients with ESRD undergoing access creation, presenting with prior central lines, pacemakers, or arm surgery (fistulas or grafts), were more likely to undergo multiple surgeries to obtain a functional graft or fistula for hemodialysis (chi-square $[1, N=53]$ = exact $P=.04$ ) than patients with ESRD presenting for access creation without prior central lines, pacemakers, or prior arm surgery. No significance was found between comorbidities DM/HTN/CAD alone or grouped as cohort and multiple surgeries (chi-square $[1, N=53]=$ exact $P>.05)$. This is somewhat surprising because patients with HTN and DM are prone to intima-media hyperplasia and microcalcifications, which may preclude a fistula from maturing. ${ }^{16}$ The suggestive correlation between multiple surgeries and prior fistula, graft, or central/line pacemaker supports the objective for development of a formal preoperative plan at the study site medical center using improved preoperative workup. The literature supports duplex ultrasound use as part of preoperative evaluation. Gray-scale ultrasound is conventional B-mode (black and white) that can identify only structural dimensions of vessel anatomy. Duplex ultrasound combines conventional gray-scale ultrasound, adding color scale duplex, which displays blood flow velocity and direction within the vessel being studied. ${ }^{6}$ In addition, duplex ultrasound can identify potential stenotic areas that may 
progress to thrombosis and failure of the AVF by identifying the peak systolic velocity and systolic velocity ratio that increase in the presence of stenosis.11, 12, 13

Additional findings from this study reinforce the need for timely referral for access evaluation. Of the total sample, $43.4 \%$ had no prior fistula or graft, yet $92.5 \%$ presented in CKD stage 5 , which is the stage when most patients start hemodialysis. Patients in stage 4 CKD should be referred to a vascular surgeon for preoperative planning with an AVF to be constructed as soon as possible so the fistula may be ready for use when hemodialysis is begun. With increasing health care costs and the implications of central line infections, early referral is imperative. The US Renal Data System 2010 report cites the per person per year (2008) total cost for patients with ESRD with a catheter as $\$ 90,110$, for an AV graft as $\$ 79,337$, and for an AVF as $\$ 64,701 .{ }^{25}$ The KDOQI 2006 guidelines recommend referral for AVF placement as the initial option for access approximately 6 months before the need for chronic hemodialysis. $^{3}$

\section{Limitations and implications}

A retrospective review has potential limitations regarding internal validity, including confounding variables, selection bias, researcher bias, maturation (changes over time), and instrumentation or changes in the way data are collected. ${ }^{19}$ These potential limitations were minimized by a single investigator collecting data at 1 site. Data were easily accessed via uniform electronic records. All patients in the 13-month study period were included, which assisted in limiting selection or researcher bias. The small sample size and the retrospective nature in general are limiting factors. A prospective longitudinal study would enable subjects to be followed for evaluation of maturation (6-8 weeks after fistula creation), central line complications, and frequency rates of functional access at time of hemodialysis. The Centers of Medicare and Medicaid Services national initiative Fistula First has set goals of increasing the use of AVFs to $\geq 50 \%$ for new patients and $\geq 66 \%$ for those already on hemodialysis. According to this initiative, only approximately $41 \%$ of patients on hemodialysis nationwide dialyze with a fistula. ${ }^{24}$ The precise percentage of grafts or fistulas available for functional use via hemodialysis was not obtained because of the retrospective nature of the study, although $21 \%$ of subjects were identified as having complications during the study period. This would disqualify them as having a functional access for hemodialysis. External validity regarding the generalizability of results also is a potential limitation. Further research into other vascular access programs would lend support to the study's results.

\section{Conclusions}

The establishment of a formal preoperative protocol using duplex ultrasound should be explored for patients identified in the KDOQI guidelines and supported in the retrospective review. To influence a change in practice, an association between the evidence and the problem must be the initial step. The primary objective to identify those potential cases that may indicate the need for a more extensive preoperative workup was met. The current preoperative assessment used for identifying fistula sites in patients with ESRD may need to be revised, particularly when additional information may be needed to identify sites that are potentially problematic, particularly in those with prior central lines, fistulas, or grafts. 
A vital component to an efficacious vascular access program is the use of a multidisciplinary approach, including nephrologists, surgeons, nurses, nurse practitioners, and interventional radiologists. Careful preoperative planning, including early referral, thorough vessel evaluation, and selection of suitable surgical site, is crucial for an optimally functioning mature access. Patients identified as appropriate should have duplex ultrasound incorporated into their preoperative planning. Nursing staff working in renal dialysis would be able to rely on access fistulas or grafts with the greatest potential for longevity and successful maturation. Further collaboration with the nephrology nurse practitioner would enhance the preoperative workup before access planning. Policy modification using duplex ultrasound should be explored as a practice standard for the study center.

The protocol could be disseminated among the nursing dialysis staff, nephrologists, and nephrology and vascular nurse practitioners, and become incorporated into the standard initial consultation workup. The patients identified via the protocol would then have duplex ultrasound ordered and complete on initial referral. Valuable time will be saved with an appropriate surgical plan that could in turn reduce the frequency of revision surgeries. This would reduce the costs of revision surgery and central line infection rates, and enable the dialysis nursing staff to have consistent, reliable hemodialysis access. Dependable vascular access, with the potential for successful maturation, will provide immediate access to hemodialysis and less reliance on central lines and their associated morbidity.

\section{References}

1 S.Q. Lew, K. Kaveh. Dialysis access related infections. ASAIO J, 46 (2000), pp. S6-S12

2 A.J. Freeman, M. Gallagher, A. Gray-Weale, E. Lippey, P. Thursby. Surgical practice to reduce dialysis access insufficiency. ANZ J Surg, 78 (2008), pp. 377-382

3 National Kidney Foundations Kidney Disease Outcomes Quality Initiative Guidelines. (2006). Clinical practice guidelines for vascular access, update 2006. Available from: http://www.kidney.org/professionals/KDOQI/guideline_upHD_PD_VA/index.htm. Accessed August 29, 2012.

$4 \mathrm{M}$. Malovrh. Strategy for the maximal use of native arteriovenous fistulae for hemodialysis ScientificWorldJournal, 6 (2006), pp. 808-815

5 M. Malovrh. Native arteriovenous fistula: preoperative evaluation. Am J Kidney Dis, 39 (2002), pp. 1218-1225

6 I. Davidson, D. Chan, B. Dolmatch, et al. Duplex ultrasound evaluation for dialysis access selection and maintenance: a practical guide. J Vasc Access, 9 (2008), pp. 1-9

7 R.N. Planken, X.H. Keuter, A.P. Hoeks, et al. Diameter measurements of the forearm cephalic vein prior to vascular access creation in end-stage renal disease patients: graduated pressure cuff versus tourniquet vessel dilatation. Nephrol Dial Transplant, 21 (2006), pp. 802-806

8 R.P. Newhouse, S.L. Dearholt, S.S. Poe, L.C. Pugh, L.M. White. Johns Hopkins nursing evidence-based practice model and guidelines. Sigma Theta Tau International, Indianapolis (IN) (2007)

9 A. Pearson, J. Field, Z. Jordan. Evidence-based clinical practice in nursing and health care. Malden (MA), Blackwell (2007) 
10 C. Glass, M. Johansson, W. DiGragio, K.A. Illig. A meta-analysis of preoperative duplex ultrasound vessel diameters for successful radiocephalic fistula placement. J Vasc Ultra, 33 (2008), pp. 6568

11 L. Salman, M. Ladino, M. Alex, et al. Accuracy of ultrasound in the detection of inflow stenosis of arteriovenous fistulae: results of a prospective study. Semin Dial, 23 (2010), pp. 117-121

12 J. Grogan, M. Castilla, L. Lozanski, A. Griffin, F. Loth, H. Bassiouny. Frequency of critical stenosis in primary arteriovenous fistulae before hemodialysis access: should duplex ultrasound surveillance be the standard of care? J Vasc Surg, 41 (2005), pp. 1000-1006

13 C.L. Ives, J.A. Akoh, J. George, E. Vaughan-Huxley, H. Lawson. Pre-operative vessel mapping and early post-operative surveillance duplex scanning of arteriovenous fistulae. J Vasc Access, 10 (2009), pp. 37-42

14 M. Allon, S. Litovsky, C.J. Young, et al. Medial fibrosis, vascular calcification, intimal hyperplasia, and arteriovenous fistula maturation. Am J Kidney Dis, 58 (2011), pp. 437-443

15 E. Korten, I.M. Toonder, Y.C. Schrama, W.C. Hop, A.C. van der Ham, C.H. Wittens. Dialysis fistulae patency and preoperative diameter ultrasound measurements. Eur J Vasc Endovasc Surg, 33 (2007), pp. 467-471

16 Y.M. Ku, Y.O. Kim, J.I. Kim, et al. Ultrasonographic measurement of intima-media thickness of radial artery in pre-dialysis uraemic patients: comparison with histological examination. Nephrol Dial Transplant, 21 (2006), pp. 715-720

17 L.S. Lauvao, D.M. Ihnat, K.R. Goshima, L. Chavez, A.C. Gruessner, J.L. Mills Sr. Vein diameter is the major predictor of fistula maturation. J Vasc Surg, 49 (2009), pp. 1499-1504

18 R. Roca-Tey, A. Rivas, R. Samon, O. Ibrik, R. Martinez Cercos, J. Viladoms. Noninvasive assessment of forearm vessels by color Doppler ultrasonography (CDU) before and after radiocephalic fistula (RCF) placement. Nefrologia, 27 (2007), pp. 489-495

19 A. Gawlinski, D. Rutledge. Selecting a model for evidence-based practice changes: a practical approach. AACN Adv Crit Care, 19 (2008), pp. 291-300

20 D.F. Polit, C.T. Beck. Nursing research: generating and assessing evidence for nursing practice. Wolters Kluwer Health/Lippincott Williams \& Wilkins, Philadelphia (PA) (2012)

21 M.G. Titler, C. Kleiber, V.J. Steelman, et al. The lowa model of evidence-based practice to promote quality care. Crit Care Nurs Clin North Am, 13 (2001), pp. 497-509

$22 \mathrm{~K}$. Reavy, S. Tavernier. Nurses reclaiming ownership of their practice: implementation of an evidence-based practice model and process. J Contin Educ Nurs, 39 (2008), pp. 166-172

23. F. Faul, E. Erdfelder, A. Buchner, A.G. Lang. Statistical power analyses using G*Power 3.1: tests for correlation and regression analyses. Behav Res Methods, 41 (2009), pp. 1149-1160

24 U.S. Department of Health and Human Services, Centers for Medicare and Medicaid Services. (2011). End Stage Renal Disease (ESRD) Quality Initiative: Fistula First Breakthrough. Available from: http://www.cms.gov/ESRDQualitylmprovelnit/04_FistulaFirstBreakthrough.asp. Accessed August 29, 2012.

25 U.S. Renal Data System, USRDS 2010 Annual Data Report: Atlas of Chronic Kidney Disease and EndStage Renal Disease in the United States, National Institutes of Health, National Institute of Diabetes and Digestive and Kidney Diseases, Bethesda, MD; 2010; Available from: http://www.usrds.org/atlas.htm. Accessed August 29, 2012. 
Conflicts of Interest: None.

This manuscript has not been presented at any conference or meetings, or submitted to any other journal. Institutional review board approval was obtained from the Zablocki VA Medical Center and Marquette University. 